\title{
The Genetic Overlap of Attention-Deficit/Hyperactivity Disorder and Autistic-like Traits: an Investigation of Individual Symptom Scales and Cognitive markers
}

\author{
Rebecca Pinto $^{1}$ • Fruhling Rijsdijk ${ }^{1}$ - Angelica Ronald ${ }^{2}$ • Philip Asherson ${ }^{1}$. \\ Jonna Kuntsi ${ }^{1}$
}

Published online: 30 May 2015

(C) Springer Science+Business Media New York 2015

\begin{abstract}
Attention-deficit/hyperactivity disorder (ADHD) and autism spectrum disorders (ASDs) frequently co-occur. However, due to previous exclusionary diagnostic criteria, little is known about the underlying causes of this covariation. Twin studies assessing ADHD symptoms and autistic-like traits (ALTs) suggest substantial genetic overlap, but have largely failed to take into account the genetic heterogeneity of symptom subscales. This study aimed to clarify the phenotypic and genetic relations between ADHD and ASD by distinguishing between symptom subscales that characterise the two disorders. Moreover, we aimed to investigate whether ADHD-related cognitive impairments show a relationship with ALT symptom subscales; and whether potential shared cognitive impairments underlie the genetic risk shared between the ADHD and ALT symptoms. Multivariate structural equation modelling was conducted on a population-based sample of 1312 twins aged 7-10. Social-communication ALTs correlated moderately with both ADHD symptom domains (phenotypic correlations around 0.30 ) and showed substantial genetic overlap with both inattention and hyperactivityimpulsivity (genetic correlation $=0.52$ and 0.44 , respectively). In addition to previously reported associations with ADHD
\end{abstract}

The Study of Activity and Impulsivity Levels in children (SAIL) was funded by a Wellcome Trust grant (GR070345MF). TEDS is supported by a program grant (G0901245; and previously G0500079) from the U.K. Medical Research Council (MRC).

Jonna Kuntsi

jonna.kuntsi@kcl.ac.uk

1 King's College London, MRC Social, Genetic and Developmental Psychiatry Centre, Institute of Psychiatry, Psychology, and Neuroscience, De Crespigny Park, SE5 8AF London, UK

2 Department of Psychological Sciences, Birkbeck, University of London, London, UK traits, reaction time variability (RTV) showed significant phenotypic $(0.18)$ and genetic $(0.32)$ association with socialcommunication ALTs. RTV captured a significant proportion $(24 \%)$ of the genetic influences shared between inattention and social-communication ALTs. Our findings suggest that social-communication ALTs underlie the previously observed phenotypic and genetic covariation between ALTs and ADHD symptoms. RTV is not specific to ADHD symptoms, but is also associated with social-communication ALTs and can, in part, contribute to an explanation of the co-occurrence of ASD and ADHD.

Keywords Attention-deficit/hyperactivity disorder(ADHD) . Autism spectrum disorders (ASDs) · Autistic-like traits $($ ALTs $) \cdot$ Reaction time variability $(\mathrm{RTV}) \cdot$ Genetics

A high co-occurrence of attention-deficit/hyperactivity disorder (ADHD) and autism spectrum disorders (ASDs) has been indicated by previous research (Rommelse et al. 2010; Simonoff et al. 2008). Despite this, previous diagnostic exclusionary criteria in the Diagnostic and Statistical Manual of Mental Disorders (DSM-IV-TR; American Psychiatric Association 2000) prohibited a dual diagnosis, although this has been amended in the most recent diagnostic revision (DSM-5; American Psychiatric Association 2013). Partly as a result of the diagnostic exclusionary criteria, little is known about the underlying causes of the covariation of these disorders (Ronald et al. 2008).

Both ADHD and autism symptoms can be viewed as continuously distributed traits (Chen et al. 2008; Dawson et al. 2002; Lubke et al. 2009; Robinson et al. 2011). Consequently, twin studies have explored shared and specific etiological influences of quantitative assessments of autistic-like traits (ALTs) and ADHD symptoms (reviewed by Posthuma and 
Polderman 2013; Ronald and Hoekstra 2011). Analysis of ratings on a UK population-based twin sample (Twins' Early Development Study; TEDS) at age 8 yielded significant phenotypic correlations ( $r \mathrm{PH}$ around 0.50$)$ between ADHD symptoms and ALTs (Ronald et al. 2008). Substantial common genetic influences (genetic correlations; $r \mathrm{G}>0.50$ ) were found whether assessing co-variation throughout the population, at the quantitative extreme, or adopting a categorical approach (Ronald et al. 2008). These findings were consistent across genders and informants. A similarly high genetic correlation ( $r \mathrm{G}=0.72$ ) was obtained for self-report symptom ratings in adulthood (Reiersen et al. 2008), and moderate to high genetic overlap has been reported in a handful of other twin studies of children and adults (Constantino et al. 2003; Lundstrom et al. 2011; Taylor et al. 2013). Taken together, these findings suggest that ALTs and ADHD symptoms are modestly correlated and share, in part, a common genetic etiology.

Yet the substantial genetic heterogeneity observed within the symptom subscales of ASD (Dworzynski et al. 2009; Robinson et al. 2012; Ronald et al. 2005, 2006a, b, 2011) and modest genetic overlap of ADHD symptom domains (Greven et al. 2011; McLoughlin et al. 2007) requires further detailed analysis. This issue was partly addressed in a sample of 2-year-old twins that separated ALTs into social and nonsocial symptom subscales (Ronald et al. 2010b). Despite the young age of the sample, the phenotypic and genetic covariation of ADHD symptoms and ALTs was evident, although slightly lower than observed in the aforementioned studies, suggestive of a possible developmental increase. Moreover, both ALT subscales contributed equally to the phenotypic covariation and etiological influences shared with ADHD symptoms (Ronald et al. 2010b). More recently, two large population-based Swedish studies of child (Ronald et al. 2014) and adult (Polderman et al. 2014) twins, focusing on parent- and self-ratings, respectively, both found that repetitive and restricted behavior and interests (versus socialcommunication difficulties) were driving the genetic overlap equally with both inattention and hyperactivity-impulsivity. A recent report from the UK TEDS sample based on parental ratings at age 12 reported greatest phenotypic and genetic overlap between autistic-like communication difficulties with both ADHD symptom scales, but also significant overlap between both ADHD symptom subscales and repetitive and restricted behavior (Taylor et al. 2015).

Aspects of executive functioning (EF) are compromised in both disorders, and have been identified as potential shared endophenotypes (Rommelse et al. 2011). In ADHD, response inhibition (as measured, for example, with commission errors (CE) on go/no-go tasks) is commonly impaired (Kuntsi et al. 2010; Willcutt et al. 2005). Although response inhibition deficits have also been reported in ASD, the evidence is more mixed (Happe et al. 2006; Nyden et al. 1999; Raymaekers et al. 2007). In two studies comparing across ADHD, ASD, and combined (ADHD $+\mathrm{ASD})$ groups, the combined group was significantly more impaired on response inhibition, compared to the ASD-only group (Buhler et al. 2011; Sinzig et al. 2008).

Another candidate for a shared cognitive impairment between ADHD and ASD is reaction time variability (RTV), thought to reflect attentional lapses. In ADHD research RTV has emerged as one of the strongest cognitive endophenotypes, indexing a substantial proportion of the genetic influences on the disorder (Kuntsi and Klein 2012). Comparisons between ADHD and ASD on RTV have to date produced a mixed set of findings, however, with reports of increased RTV in ADHD only (Johnson et al. 2007), in ASD only (Geurts et al. 2008), in both (Nyden et al. 2010) or neither disorder (Geurts et al. 2004; Raymaekers et al. 2007). More recently, when comparing children from single and comorbid diagnosis groups, increased RTV was exhibited in groups with ADHD symptoms (ADHD-only and ADHD+ASD group) versus groups with no ADHD symptoms (ASD-only and controls) (Tye et al. 2014).

In previous research on ADHD we have shown the etiological separation of RTV and CE (Kuntsi et al. 2010, 2014) and further showed in a population twin sample that these two cognitive impairments display partially distinct phenotypic and genetic relationships to the two ADHD symptom domains (Kuntsi et al. 2014). We found a strong genetic overlap between RTV and particularly inattention. CE showed less differentiation between the ADHD symptom domains, although the genetic correlations were overall low. Given these findings and the genetic heterogeneity of ALTs (Dworzynski et al. 2009; Robinson et al. 2012; Ronald et al. 2005, 2006a, b, 2011), we extended the previous model with an aim to investigate if the phenotypic and genetic covariation between ADHD symptoms and ALTS are driven by specific symptom subscales, and if cognitive impairments (CE and RTV) represent unique etiological pathways for ADHD symptoms or are shared with ALTs, and similarly show different patterns of co-occurrence across ALT subscales. Using a population-based twin sample our study aimed, specifically, to investigate: (1) to what extent are socialcommunication and non-social ALTs phenotypically and genetically associated with the two ADHD symptom domains of inattention and hyperactivity-impulsivity in childhood; (2) to what extent are social-communication and non-social ALTs phenotypically and genetically associated with RTV and CE; and (3) for any significant genetic correlations that emerge in (2), to estimate the extent to which this shared cognitive impairment underlies the genetic risk shared between ADHD symptoms and ALTs. 


\section{Methods}

\section{Sample and Procedure}

Participants were from the Study of Activity and Impulsivity Levels in children (SAIL). Sampling methods and data collection procedures are described in detail elsewhere (Kuntsi et al. 2006). The parents of all participating children provided informed consent, with ethical approval obtained from the Research Ethics Committee of the Institute of Psychiatry, King's College London, UK. The final sample consisted of 1312 children: 255 identical (monozygotic; MZ) twin pairs, 184 same-sex non-identical (dizygotic; DZ) twin pairs, and 206 opposite-sex DZ twin pairs, and 22 singletons coming from pairs with one of the twins excluded.

Twin zygosity was determined using a parental-report questionnaire with $95 \%$ accuracy, later verified using DNA (Price et al. 2000). The mean age of participating children was 8.83 years $(\mathrm{SD}=0.67)$, with a similar proportion of boys $(49.5 \%)$ and girls. Children's IQs ranged from 70 to 158 (mean=109.34, $\mathrm{SD}=14.72)$.

\section{Measures}

Rating Scales Parents and teachers were asked to complete the Long Versions of Conners' Parent and Teacher Rating Scales (Conners et al. 1998a, b). From both scales, we used the nine-item inattention and nine-item hyperactivity-impulsivity DSM-IV ADHD symptom subscales. Inter-rater agreement for parent and teacher ratings was moderate for both inattention $(r=0.45, p<0.001)$ and hyperactivity-impulsivity $(r=0.40, p<0.001)$. Parent and teacher ratings on corresponding subscales were summed to obtain a composite measure, in order to enable comparison to our previous analyses (Kuntsi et al. 2014) and to capture a more stable, reliable and situationally pervasive measure of problem behaviors. An overall inattention and hyperactive-impulsive subscale score was available for 1159 SAIL participants.

Autistic-like traits (ALTs) were rated by parents and teachers when children were aged seven via postal questionnaire. ALT parental and teacher ratings were collected, on average, respectively 18 and 15 months earlier than ADHD ratings and cognitive testing. The questionnaire was comprised mainly of behaviors that would be observed in the general population, so items relating to rare behaviors were not included (Ronald et al. 2005). The majority of items (Table 1) were derived from DSM-IV autism criteria, and divided according to the criteria as measures of socialcommunication and non-social ALTs (Ronald et al. 2005, 2010a). The 10 items in the social-communication subscale assess peer interactions, social insight, nonverbal behaviors, and unusual communication style. The six items in the nonsocial subscale assess obsessive and repetitive behaviors,
Table 1 Items used to measure autistic-like traits by socialcommunication and non-social autistic-like trait subscales

Social-communication autistic-like trait subscale

Has unusual eye gaze, facial expression or gestures

Considerate of other people's feelings (reversed)

Rather solitary or tends to play alone

Has odd style of communication; old-fashioned, formal or pedantic

Generally liked by other children (reversed)

Can take hints and keep secrets, can be discreet (reversed)

Often says things that are embarrassing for others, without realising

Gets on better with adults than with other children

Is afraid of social situations

Has at least one good friend (reversed)

Non-social autistic-like trait subscale

Is extremely distressed by changes to routine or familial arrangements

Notices small details others might miss

Insists on doing something over and over so that it interferes with day to day life

Tends to check that some things are done exactly 'right'

Fussy or over particular

Has a strong interest in an unusual topic

detail-focused behaviors and restricted interests. Inter-rater correlations were modest, but significant $(p<0.001)$, for both social-communication $(r=0.20)$ and non-social $(r=0.17)$ autistic-like subscales. However, correlations between the composite scores and individual informant scores (e.g., parent ratings for social-communication ALTs and composite socialcommunication ALTs) were significant and high (0.72-0.82).

ALT ratings were not obtained for all TEDS cohorts, and were subsequently missing for a proportion of SAIL participants. Parent and teacher ratings were summed providing an overall social-communication and non-social subscale score, respectively, for 959 and 961 SAIL participants. Participants with incomplete data were included, as Mx handles missing data by using raw maximum likelihood estimation to calculate a likelihood statistic for each observation based on the observed variance/covariance.

Wechsler Intelligence Scales for Children, Third Edition (WISC-III) (Wechsler 1991). The vocabulary, similarities, picture completion and block design subtests from the WISC-III were used to obtain an estimate of the child's IQ (prorated; see Sattler 1992 for description of procedures).

The Go/No-Go Task (Borger and van der Meere 2000; Kuntsi et al. 2005; van der Meere et al. 1995). On each trial, one of two possible stimuli appeared for $300 \mathrm{~ms}$ in the middle of the computer screen. The child was instructed to respond only to the go stimuli and to react as quickly as possible, but to maintain a high level of accuracy. The proportion of $g o$ stimuli to no-go stimuli was 4:1. The participants performed the task 
under three conditions (slow, fast and incentive), matched for length of time on task. Herein we present data from the slow condition, which had an inter-stimulus interval of $8 \mathrm{~s}$ and consisting of 72 trials, and the fast condition, with an interstimulus interval of $1 \mathrm{~s}$ and consisting of 462 trials. The order of presentation of the slow and fast conditions varied randomly across participants. We focus here on three variables obtained for the task: mean reaction time (MRT), reaction time variability (RTV, standard deviation of RTs) and commission errors (CE).

The Fast Task (Andreou et al. 2007; Kuntsi et al. 2005, 2006). The baseline condition, with a foreperiod of $8 \mathrm{~s}$ and consisting of 72 trials, followed a standard warned four-choice RT task. Four empty circles (warning signals, arranged sideby-side) first appeared for $8 \mathrm{~s}$, after which one of them (the target) was coloured in. Participants were asked to press the response key that directly corresponded in position to the location of the target stimulus. Following a response, the stimuli disappeared from the screen and a fixed inter-trial interval of $2.5 \mathrm{~s}$ followed. Speed and accuracy were emphasised equally. If the child did not respond within $10 \mathrm{~s}$, the trial terminated. A comparison condition with a fast event rate $(1 \mathrm{~s})$ and incentives followed the baseline condition (Andreou et al. 2007). Herein we focus on MRT and RTV, obtained from the baseline condition, which measures RT performance under an unrewarded, slow condition.

Selection of Cognitive Variables for Analyses To limit the total number of variables and to enable a comparison to our previous analyses (Kuntsi et al. 2010, 2014) summed scores were obtained across two tasks or conditions as follows: unstandardized RTV across fast task baseline condition and go/ no-go task slow condition; and percentage of CE across go/ no-go task slow and fast conditions. Our previous research has shown that combining cognitive phenotypes that are theoretically related (such as derived from similar tasks) creates psychometrically robust variables by improving measurement reliability (Kuntsi et al. 2006). Moreover, we have previously shown in a clinical ADHD sibling-pair sample that combining RTV across these tasks and CE across these conditions capture two familial cognitive impairment factors in ADHD, and that the cross-task and cross-condition measures used to generate the composite measures in this sample largely capture $(r \mathrm{~F}=$ 0.75 and 0.73 ) the same familial etiology (Kuntsi et al. 2010).

Summed variables were regressed to correct for the effects of age and sex (a standard twin modelling procedure) and the residuals used in analysis. Cognitive variables were further regressed for IQ. Although our previous analyses indicated that the majority of genetic influences shared between ADHD and cognitive variables were independent of those shared with IQ (Wood et al. 2010, 2011), regressing for IQ ensured we controlled for any small mediating effects of IQ that were not the focus of present analyses, consistent with our previously adopted approach (Kuntsi et al. 2010, 2014).

\section{Statistical Analyses}

Overview of the Twin Method Biometrical genetic modelling is based on three assumptions: (1) MZ twins share $100 \%$ of their segregating alleles and DZ twins share on average $50 \%$ of additive genetic (A) influences, but only $25 \%$ of non-additive genetic influences (D); (2) for twin pairs reared together, the covariance of both members of MZ and DZ twin pairs will be due to perfectly correlated shared environmental (C) influences; and (3) individual-specific environmental factors (E; which subsume any measurement error) do not contribute to the similarity between twin pairs. From this we can derive the following within-pair twin correlation expectations: (1) additive genetic influences (A) will double the MZ twinpair correlation in relation to the DZ twin-pair correlation; (2) non-additive genetic influences (D) will more than double the MZ twin-pair correlation in relation to the DZ twin-pair correlation; (3) shared environmental effects (C) will increase within-pair MZ and DZ correlations to the same extent, reflected by $\mathrm{DZ}$ correlations that are more than half of $\mathrm{MZ}$ correlations; and (4) non-shared environment (E) will decrease both $\mathrm{MZ}$ and $\mathrm{DZ}$ correlations, most commonly identified in MZ correlations that are less than 1. These predictions are used to estimate the components from observed differences in $\mathrm{MZ}$ and $\mathrm{DZ}$ correlations. In multivariate genetic analyses, as well as partitioning the phenotypic variance of single traits, the covariance between traits is decomposed into $\mathrm{A}, \mathrm{C} / \mathrm{D}$ and $\mathrm{E}$ influences following exactly the same logic as above and using the ratio of MZ:DZ differences in cross-twin cross-trait (e.g., inattention ratings in twin 1 with RTV scores in twin 2) correlations (Rijsdijk and Sham 2002).

Genetic Structural Equation Models With the exception of CE (skew: -0.12), all residual summed scores were positively skewed (1.06 to 1.92) and were transformed to approximate a normal distribution (using the optimised minimal skew command in STATA version 9; Stata 2007). Genetic structural equation modelling was conducted in the program $\mathrm{Mx}$ (Neale et al. 2006).

Saturated Phenotypic Model Before genetic modelling, to obtain twin correlations, to test assumptions of the genetic method (i.e., equality of means and variances across twins and zygosity groups) and to provide a baseline comparison for subsequent genetic models, a saturated model was fitted that fully describes the data using the maximum number of free parameters, modelling the observed means and variances without dissecting variance or covariance into etiological components. 
Parameter Selection for Multivariate Genetic Analyses Univariate genetic analyses (not presented) showed an ACE model to provide the best fit for cognitive measures, while an ADE model (with scalar sex differences) fitted the subscale ratings best. However, to deal with limited power to distinguish between $\mathrm{A}$ and $\mathrm{D}$ effects and to accommodate finding the overall (broad-sense) genetic overlap $(\mathrm{G})$ between all traits, we fitted an AE model for subscale ratings and an ACE model for cognitive measures. This multivariate model included a scaling factor to account for male and female variance differences. No other quantitative and qualitative sex differences in genetic parameters were indicated.

Correlated Factor Solution of the Full Cholesky Decomposition Model (Fig. 1) The main multivariate genetic model used is descriptive and applies a triangular decomposition for each modelled etiological factor. The solution is then converted to the mathematical equivalent correlated factors model (Loehlin 1996), in which the order of traits is arbitrary. The mathematical solution describes the degree of overlapping etiological factors between two traits, with etiological correlations that vary from 0 (indicative of no overlap) to 1 (reflecting complete overlap), as well as standardized components for each variable (Fig. 1).

Cholesky Decomposition Model (Fig. 2) In the Cholesky, a triangular decomposition is used, to decompose the variance in each phenotype and covariance between the phenotypes into $\mathrm{A}, \mathrm{D} / \mathrm{C}$ and $\mathrm{E}$ influences. The ordering of the traits in the Cholesky model was decided a priori: to ascertain how much of the etiological overlap between ADHD and ALT symptom subscales were shared with cognitive measures. As such, for these analyses we present the triangular (Cholesky) decomposition with a reduced number of variables and cognitive impairment assigned as the first measured variable (Fig. 2).

\section{Results}

Given the variance differences between the genders, means and standard deviations are presented separately for males and females (Table 2).

The focus of this paper is on the covariance of socialcommunication and non-social ALTs each with ADHD symptom subscales and cognitive variables. Accordingly, in Table 3 we present maximum likelihood cross-twin cross-trait correlations for the ALT subscales separately with each of the remaining variables. Similarly in Table 4, we present parameter estimates for the specific relationships of socialcommunication and non-social ALTs with behavioural ADHD and cognitive variables (for all parameter estimates between all the variables, see Fig. 1).
Social-Communication and Non-Social ALTs With the two ADHD Symptom Domains of Inattention and Hyperactivity-Impulsivity

Social-communications ALTs correlated moderately and equally with both inattention $(r \mathrm{PH}=0.33 ; 95 \%$ confidence intervals (CI) $0.26-0.39$ ) and hyperactivity-impulsivity $(r \mathrm{PH}=0.31 ; 0.24-0.37)$. In contrast, the correlation between non-social ALTs and hyperactivity-impulsivity was significantly (non-overlapping CI) lower $(r \mathrm{PH}=0.11 ; 0.04-0.18)$ and did not reach significance with inattention. The phenotypic covariance between social-communication ALTs and inattention was predominantly accounted for by shared broadsense genetic effects $(96 \%)$. Although the proportion of the phenotypic covariance between social-communication ALTs and hyperactivity-impulsivity, and non-social ALTs and either ADHD behavioral dimension, could not be quantified (etiological correlations had both positive and negative values), visual inspection of raw estimates (Table 4) suggested that the majority of the phenotypic correlations were due to shared genetic influences.

The broad-sense genetic correlations for socialcommunication ALTs were substantial (Table 4) and showed little differentiation (overlapping $\mathrm{CI}$ ) with either ADHD symptom domain (inattention $(r \mathrm{G}=0.52)$ and hyperactivityimpulsivity $(r \mathrm{G}=0.44))$. The genetic correlation between non-social ALTs and hyperactivity-impulsivity was significantly lower $(r \mathrm{G}=0.20)$. The broad-sense genetic correlation between non-social ALTs and inattention was low and nonsignificant.

\section{Social-Communication and Non-Social ALTs With RTV and $\mathrm{CE}$}

Social-communications ALTs were significantly correlated with RTV $(r \mathrm{PH}=0.18)$, with broad-sense genetic effects accounting for the vast majority $(87 \%)$ of the phenotypic covariation (Table 4). The broad-sense genetic correlation between RTV and social-communication ALTs was moderate $(r \mathrm{G}=0.32)$. Non-social ALTs showed a small but significant negative correlation with $\mathrm{CE}(r \mathrm{PH}=-0.09)$. There were no significant correlations seen between RTV and non-social ALTs, or between CE and social-communications ALTs (Table 4).

\section{Cholesky Decomposition: Social-communication ALTs, Inattention and RTV}

In the full correlated factors solution (Fig. 1), inattention and social-communication ALTs showed strong overlapping broad-sense genetic effects $(r \mathrm{G}=0.52)$ and RTV displayed substantial shared broad-sense genetic influences with both inattention $(r \mathrm{G}=0.40)$ and social-communication ALTs 


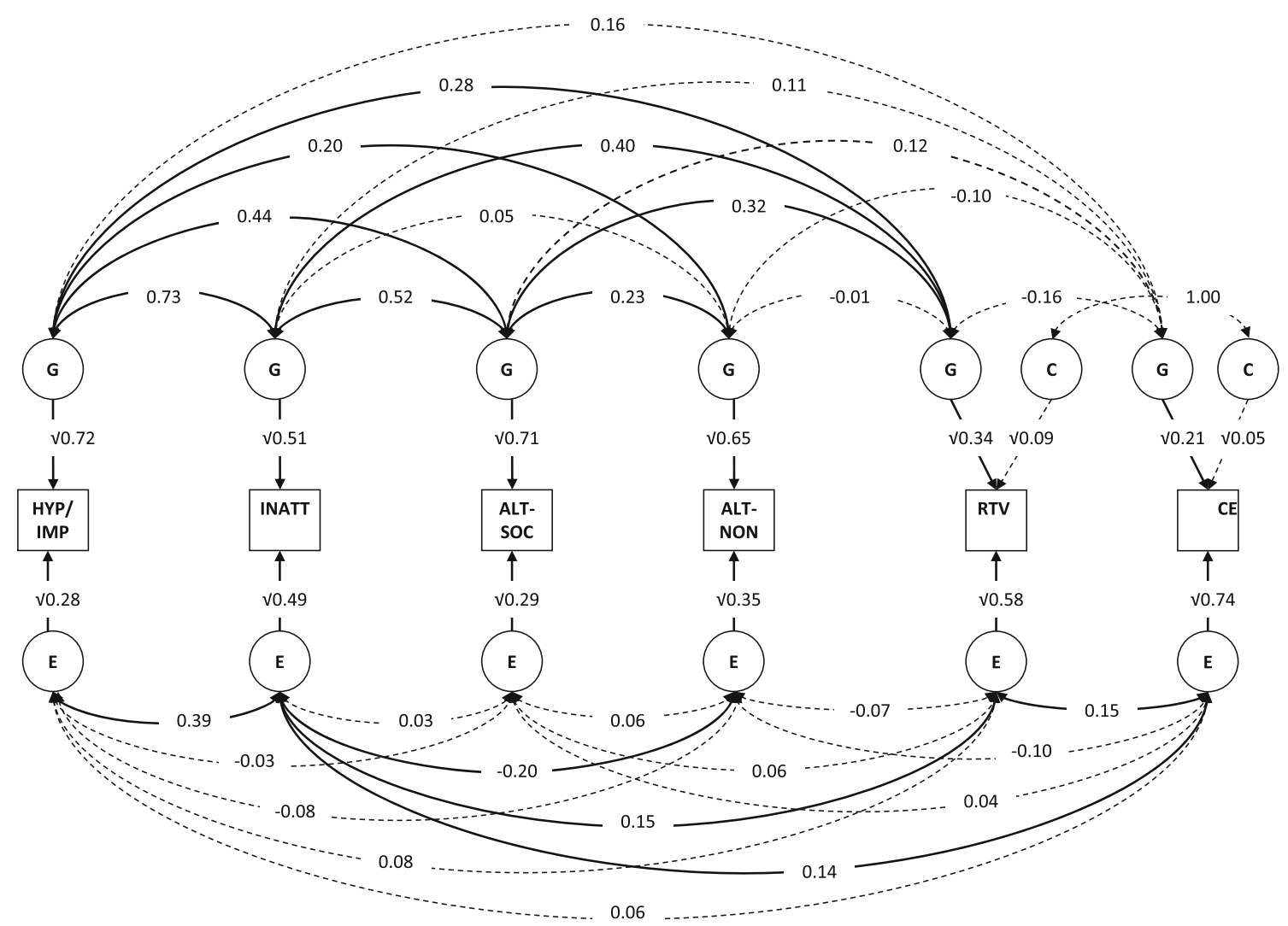

Fig. 1 Correlated factor solution of the full ACE Cholesky Decomposition. Note: Significant parameters are indicated with solid lines; non-significant parameters in dotted lines; Abbreviations: HYPIMP Hyperactivity-impulsivity, INATT inattention, ALT-SOC socialcommunication autistic-like trait subscale, ALT-NON non-social

( $r \mathrm{G}=0.32$ ). Based on these multivariate findings, we selected social communication ALTs, inattention and RTV for further investigation of their interrelationships. Therefore we selected to test in the Cholesky decomposition (Fig. 2), how much of the broad-sense genetic effects shared between inattention and social-communication ALTs were also shared with RTV. This was tested using a reduced three-factor Cholesky decomposition, with RTV assigned as the first variable, and estimated by summing the product of Cholesky genetic paths that are shared with RTV and taking them as a percentage of the total genetic covariance between inattention and socialcommunication ALTs. We also estimated how much of the covariance between social-communication ALTs and inattention was shared with individual-specific environmental risk factors shared with RTV. (Common environment (C) did not contribute to the covariation between RTV and either inattention or social-communication ALTs and so is not included in the model.)

Using the parameter estimates from the Cholesky decomposition, we estimated that $24 \%$ of the broad-sense genetic covariance between inattention and social-communication ALTs was shared with genetic effects underlying RTV: $((0.77 * 0.77)$ / autistic-like trait subscale, $R T V$ reaction time variability, $C E$ commission errors, $G$ broad-sense genetic influences, $C$ shared environmental influences, $E$ individual-specific environmental influences; Model presented for one twin only for ease of presentation

$(0.77 * 0.77)+(1.82 * 1.03)=0.59 /(0.59+1.87)=0.59 / 2.47=$ $0.24)$. In a similar vein, $57 \%$ of the individual-specific environmental covariance between social-communication ALTs and inattention was shared with RTV.

\section{Discussion}

An investigation of the individual ADHD and ALT symptom domains indicated, first, that the phenotypic and genetic overlap between ADHD symptoms and ALTs in this study is largely driven by social-communication ALTs, equally with both inattention and hyperactivity-impulsivity. Second, socialcommunication ALTs were phenotypically and genetically correlated with RTV, consistent with the view that increased RTV is not specific to ADHD symptoms (or, specifically, inattention symptoms). Third, RTV captured a significant proportion $(24 \%)$ of the genetic influences shared between inattention and social-communication ALTs.

Our study suggests that the previously observed phenotypic and genetic association between ADHD and ASD is predominantly driven by social-communication ALTs. 


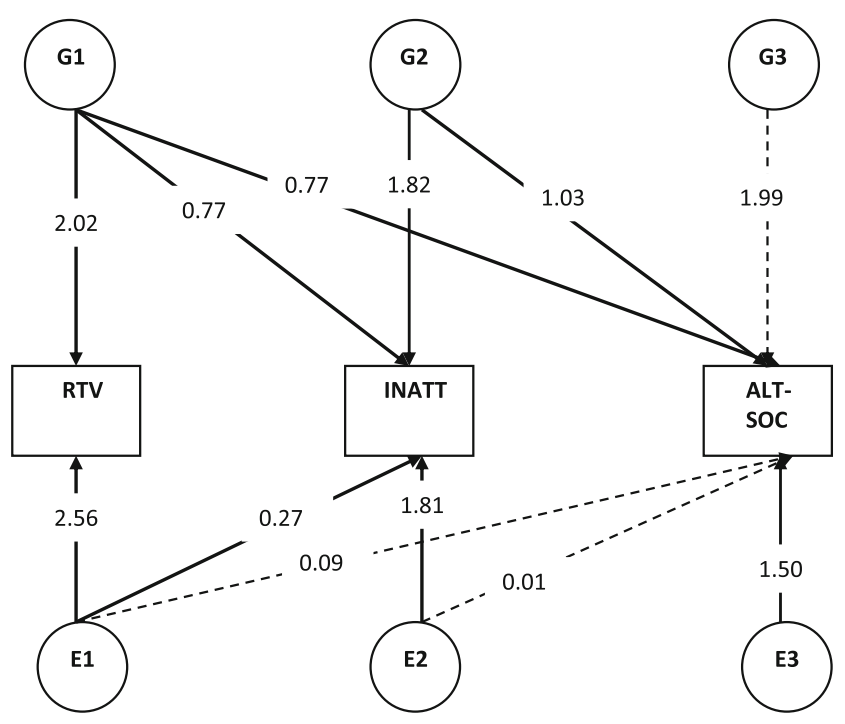

Fig. 2 Broad-sense genetic and individual-specific environmental parameter estimates from the reduced three-variable Cholesky model. Note: unstandardised parameter estimates; significant parameters are indicated with solid lines and non-significant parameters with dotted lines; Abbreviations: RTV reaction time variability, INATT inattention, $A L T-S O C$ social-communication autistic-like traits, $G$ broad-sense genetic influences, $E$ individual-specific environmental influences; Model presented for one twin only for ease of presentation and for the etiological factors that contributed to covariation between all traits (i.e., $\mathrm{G}$ and $\mathrm{E}$ )

Furthermore, inattention and hyperactivity-impulsivity are similarly associated with social-communication ALTs. The findings indicate that the phenotypic and genetic covariation between the symptoms of these two disorders is partially symptom-specific, driven by social-communication ALTs equally with both ADHD symptom subscales. This pattern of findings is partially in line with the Generalist Genes hypothesis, that the same set of genetic risk factors will influence symptom subscales across disorders: in our study, a set of generalist genes were influencing inattention, hyperactivityimpulsivity and social-communication ALTs. In addition, this pattern of findings is consistent with the greater genetic heterogeneity across ALTs (Dworzynski et al. 2009; Robinson et al. 2012; Ronald et al. 2005, 2006a, b, 2011), than across ADHD symptom dimensions (Greven et al. 2011; McLoughlin et al. 2007).

Of the previous twin studies on subscales of ADHD and ALT, two reported strongest overlap between repetitive and restricted behavior and interests and ADHD traits (Polderman et al. 2014; Ronald et al. 2014), in contrast to our results which showed strongest associations with social-communication ALTs. It is unclear why these larger studies, one on children and one on adults, reported a different pattern of results to those seen here. A third recent study of the TEDS sample reported that communication ALTs were most strongly linked with both ADHD traits, which is more similar to our findings (Taylor et al. 2015), although their social ALT scale showed low overlap with ADHD traits). It is important to note that our sample is a subset of TEDS, and so these findings are not fully independent, although Taylor's study used assessments obtained at age 12 from the Childhood Autism Spectrum Test (Scott et al. 2002). An important direction of future research is to clarify the source of the discrepant findings.

Our findings on cognitive markers are in line with the previous studies that have found that increased RTV is not specific to ADHD, but is also observed in ASD (Nyden et al. 2010). Our findings now extend these observations by indicating that the association between RTV and ASD is driven by social-communication ALTs, and by showing that genetic influences explain the majority ( $87 \%$ ) of this association. Moreover, a notable proportion $(24 \%)$ of the genetic effects shared between inattention and social-communication ALTs could be accounted for by RTV. This suggests that a modest set of shared genetic risk factors contribute to RTV, inattention and social-communication ALTs. The second cognitive marker that we investigated - response inhibition (CE) - did not emerge as meaningfully associated to ALTs, as the only phenotypic or etiological correlation that emerged as significant was a low, negative phenotypic correlation with non-social ALTs $(r \mathrm{PH}=-0.09)$. The previous findings on response inhibition in ASD groups have been mixed (Buhler et al. 2011;

Table 2 Means and standard deviations for behavioral ratings and cognitive measures

\begin{tabular}{lllllll}
\hline & $\begin{array}{l}\text { Hyperactivity-impulsivity }^{\mathrm{a}} \\
(n=1159(88 \%))\end{array}$ & $\begin{array}{l}\text { Inattention }^{\mathrm{a}} \\
(n=1159(88 \%))\end{array}$ & $\begin{array}{l}\text { Social-communication ALTS }^{\mathrm{a}}(n=959(73 \%)) \\
\text { Non-social ALTs } \\
(n=961(73 \%))\end{array}$ & $\begin{array}{l}\mathrm{RTV}^{\mathrm{b}} \\
(n=1247(95 \%))\end{array}$ & $\begin{array}{l}\mathrm{CE}^{\mathrm{c}} \\
(n=1290(98 \%))\end{array}$ \\
\hline MZM & $\mathbf{1 1 . 0 6 ( 8 . 6 1 )}$ & $\mathbf{1 2 . 7 0}(\mathbf{8 . 9 5})$ & $\mathbf{6 . 0 1}(\mathbf{3 . 5 7})$ & $\mathbf{5 . 8 6}(\mathbf{3 . 1 5})$ & $\mathbf{6 1 9 . 0 6 ( 3 5 0 . 8 1 )}$ & $\mathbf{1 1 6 . 5 2 ( 3 4 . 2 9 )}$ \\
MZF & $\mathbf{6 . 7 4 ( 5 . 8 9 )}$ & $\mathbf{7 . 7 9}(\mathbf{6 . 5 1})$ & $\mathbf{4 . 5 3}(\mathbf{2 . 9 9})$ & $\mathbf{5 . 5 8}(\mathbf{2 . 4 4})$ & $\mathbf{6 2 9 . 9 4 ( 3 6 4 . 1 5 )}$ & $\mathbf{9 6 . 4 2 ( 3 1 . 4 7 )}$ \\
DZM & $11.53(9.64)$ & $14.25(11.14)$ & $6.20(3.79)$ & $6.57(3.30)$ & $631.01(376.52)$ & $115.59(32.90)$ \\
DZF & $7.31(6.49)$ & $9.06(7.88)$ & $5.08(3.29)$ & $5.80(2.80)$ & $628.04(359.12)$ & $95.61(33.14)$ \\
\hline
\end{tabular}

$n$ number of observations, MZM monozygotic male, $M Z F$ monozygotic female, DZM dizygotic male, DZF dizygotic female, ALTs autistic-like traits, $R T V$, reaction time variability, $C E$ commission errors

${ }^{\text {a }}$ Sum of parent and teacher ratings

${ }^{\mathrm{b}}$ Sum of unstandardised data scores across fast task baseline and go/no-go slow conditions

${ }^{\mathrm{c}}$ Sum of percentages of CE across go/no-go slow and fast conditions; $\mathbf{M Z}$ data in bold typeface, DZ data in italic typeface 
Table 3 Maximum-likelihood cross-twin cross-trait correlations (constrained correlated model) for social-communication and nonsocial ALTs between ADHD symptoms and cognitive measures

\begin{tabular}{lll}
\hline Cross-trait correlations & & \\
\hline & Cross-twin (MZ) & Cross-twin (DZ) \\
\hline Hyperactivity-impulsivity with: & & \\
Social-communication ALTs & $\mathbf{0 . 3 1}(\mathbf{0 . 2 3} / \mathbf{0 . 3 7})$ & $\mathbf{0 . 1 4}(\mathbf{0 . 0 6} / \mathbf{0 . 2 2})$ \\
Non-social ALTs & $\mathbf{0 . 1 3}(\mathbf{0 . 0 5 / 0 . 1 9})$ & $0.08(-0.002 / 0.15)$ \\
Inattention with: & & \\
Social-communication ALTs & $\mathbf{0 . 3 1}(\mathbf{0 . 2 3} / \mathbf{0 . 3 5})$ & $\mathbf{0 . 1 3}(\mathbf{0 . 0 4} / \mathbf{0 . 2 1})$ \\
Non-social ALTs & $-0.01(-0.09 / 0.03)$ & $0.06(-0.02 / 0.10)$ \\
RTV with: & $\mathbf{0 . 1 6}(\mathbf{0 . 0 8} / \mathbf{0 . 2 4})$ & $0.03(-0.05 / 0.10)$ \\
Social-communication ALTs & $0.01(-0.10 / 0.05)$ & $0.01(-0.07 / 0.06)$ \\
Non-social ALTs & & $-0.01(-0.08 / 0.07)$ \\
CE with: & $0.01(-0.07 / 0.09)$ & $0.02(-0.06 / 0.08)$ \\
Social-communication ALTs & $-\mathbf{0 . 0 5}(-\mathbf{0 . 1 3} /-\mathbf{0 . 0 1})$ & \\
Non-social ALTs &
\end{tabular}

Due to the lack of quantitative and qualitative sex differences, $\mathrm{MZ}$ and $\mathrm{DZ}$ correlations are not presented by sex; $95 \%$ Confidence Intervals given in parentheses; Significant $(p<0.05)$ estimates in bold typeface; Non-significant estimates in normal typeface

$A L T s$ autistic-like traits, $R T V$ reaction time variability, $C E$ commission errors, $M Z$ Monozygotic, $D Z$ Dizygotic
Happe et al. 2006; Nyden et al. 1999; Raymaekers et al. 2007; Sinzig et al. 2008). The present study adds to this evidence by showing that response inhibition does not account for the overlap between ADHD and ALT symptoms.
The genetic overlap of RTV with symptom subscales across both ADHD and ALTs (specifically inattention and social-communication ALTs) suggest that RTV can be considered a trans-diagnostic cognitive endophenotypes. The highly heterogeneous clinical presentation, genetically complex

Table 4 Etiological and phenotypic correlations (standardised correlated factors solution genetic model) for social-communication and non-social ALTs between ADHD symptoms and cognitive measures

\begin{tabular}{|c|c|c|c|c|c|}
\hline & \multicolumn{2}{|c|}{ Etiological correlations } & \multirow{2}{*}{$\begin{array}{l}\text { Phenotypic correlations } \\
r \mathrm{PH}\end{array}$} & \multicolumn{2}{|c|}{$\begin{array}{l}\text { Contribution of covariance accounted for by } \\
\text { etiological factors }{ }^{\text {a }}\end{array}$} \\
\hline & $r \mathrm{G}$ & $r \mathrm{E}$ & & $r \mathrm{PH}-\mathrm{G}$ & $r \mathrm{PH}-\mathrm{E}$ \\
\hline \multicolumn{6}{|l|}{ Hyperactivity-impulsivity with: } \\
\hline Social-communication ALTs & $0.44(0.33 / 0.55)$ & $-0.03(-0.17 / 0.11)$ & $0.31(0.24 / 0.37)$ & $0.32 *$ & $-0.01^{*}$ \\
\hline Non-social ALTs & $0.20(0.08 / 0.32)$ & $-0.08(-0.21 / 0.07)$ & $0.11(0.04 / 0.18)$ & $0.14^{*}$ & $-0.03 *$ \\
\hline \multicolumn{6}{|l|}{ Inattention with: } \\
\hline Social-communication ALTs & $0.52(0.39 / 0.65)$ & $0.03(-0.12 / 0.18)$ & $0.33(0.26 / 0.39)$ & $0.31(96 \%)$ & $0.01(4 \%)$ \\
\hline Non-social ALTs & $0.05(-0.11 / 0.21)$ & $-0.20(-0.34 /-0.05)$ & $-0.06(-0.13 / 0.01)$ & $0.03^{*}$ & $-0.08^{*}$ \\
\hline \multicolumn{6}{|l|}{ RTV with: } \\
\hline Social-communication ALTs & $0.32(0.15 / 0.66)$ & $0.06(-0.07 / 0.19)$ & $0.18(0.11 / 0.25)$ & $0.16(87 \%)$ & $0.02(13 \%)$ \\
\hline Non-social ALTs & $-0.01(-0.20 / 0.21)$ & $-0.07(-0.20 / 0.06)$ & $-0.04(-0.11 / 0.04)$ & $-0.01(13 \%)$ & $-0.03(87 \%)$ \\
\hline \multicolumn{6}{|l|}{ CE with: } \\
\hline Social-communication ALTs & $0.12(-0.35 / 0.37)$ & $0.04(-0.09 / 0.16)$ & $0.03(-0.05 / 0.09)$ & $0.04(71 \%)$ & $0.01(29 \%)$ \\
\hline Non-social ALTs & $-0.10(-0.68 / 0.15)$ & $-0.10(-0.22 / 0.03)$ & $-0.09(-0.15 /-0.02)$ & $-0.04(42 \%)$ & $-0.05(58 \%)$ \\
\hline
\end{tabular}

$95 \%$ Confidence Intervals given in parentheses; Significant $(p<0.05)$ estimates in bold typeface; Non-significant estimates in normal typeface $A L T s$ autistic-like traits, $R T V$ reaction time variability $C E$ commission errors, $r \mathrm{G}$ broad-sense genetic correlation, $r \mathrm{E}$ individual-specific environmental correlation, $r \mathrm{PH}$ phenotypic correlation, $r \mathrm{PH}-\mathrm{G}$ phenotypic covariance due to broad-sense genetic effects, $r \mathrm{PH}-\mathrm{E}$ phenotypic covariance due to individual-specific environmental effects

*It was not possible to formally estimate these proportions, due to the presence of both positive and negative etiological correlations between relevant variables

${ }^{\text {a }}$ The contribution of etiological factors to the phenotypic correlation is given as a raw estimate, and as a percentage in brackets 
nature, and non-optimal phenotypic definition of ADHD and ASD, likely contribute to the challenges involved in identifying genetic risk markers. One way to overcome these obstacles may be to use endophenotypes as targets for molecular genetic studies or as a means of subdividing samples into more homogenous subgroups (Losh et al. 2008; Viding and Blakemore 2007), potentially across current diagnostic categories (Levy and Ebstein 2009). However, the main appeal of endophenotypes is in bridging the gap between etiological factors and clinical phenotypes by elucidating underlying pathophysiological processes (Meyer-Lindenberg 2010). Our previous findings indicated specific neurocognitive pathways, with RTV particularly underlying inattentive symptoms (Kuntsi et al. 2014). The findings in this study suggest that this gene-behavioral pathway may not be unique, but is a partly common pathway underlying social-communication ALTs.

Our findings underline that investigating the individual symptom domains of ADHD and ALTs may clarify the etiological link between these two commonly occurring disorders. Quantitative genetic studies that identify genetically-related behavioral traits, even across diagnostic boundaries, can inform the selection of additional genetic markers for candidate gene association studies. In this respect, our findings suggest that molecular genetic investigations may benefit from examining putative genetic risk markers for inattention or hyperactivity-impulsivity for social-communication ALTs, and vice-versa. The clustering of cross-diagnostic symptom profiles (see also Ronald et al. 2014) further supports the change in diagnostic criteria to allow a dual diagnosis of ADHD and ASD. However, our findings also highlight that the etiology of disorders does not necessarily follow diagnostic boundaries, and accordingly may give rise to different ways of conceptualizing disorders.

The findings presented here indicate shared genetic effects between RTV, inattention and social-communication ALTs, but cannot clarify whether a causal relationship is involved. A further limitation of our study was that the behavioral ratings on ADHD and ALT symptoms were not collected simultaneously, which will have the effect of dampening the observed phenotypic correlations. Future studies should aim to replicate our findings. In addition, RTV only partially accounts for the covariation of social-communication ALTs and inattention; therefore future studies should extend investigations to include additional cognitive markers. Finally, although there is good evidence that both ADHD and ASD represent extremes of traits that are continuously distributed throughout the population, studies of clinical samples are required before these findings can be generalised to clinical populations.

Overall, our findings on the distinction between socialcommunication and non-social ALTs in their association with ADHD symptoms emphasise the importance of analysing symptom sub-domains in the investigation of neurodevelopmental disorders. The genetic overlap we observed across some of the behavioral symptoms, as well as with the cognitive marker of RTV, is consistent with the many reports in psychiatric genetics of partly shared genetic influences across psychiatric phenotypes (Cross-Disorder Group of the Psychiatric Genomics Consortium 2013); yet we also observed a degree of specificity. Future research that incorporates additional levels of analysis, such as neurophysiological or other neuroimaging approaches, will likely help to clarify further the similarities and differences between the overlapping neurodevelopmental disorders of ADHD and ASD. The changes in diagnostic practice will allow more research into the co-occurrence of these disorders and contribute to a greater understanding of their individual and shared etiology.

Acknowledgments We gratefully acknowledge the ongoing contribution of the participants in the Twins Early Development Study (TEDS) and their families. We thank the TEDS-SAIL families, who give their time and support so unstintingly. We also thank research team members Keeley Brookes, Rebecca Gibbs, Hannah Rogers, Eda Salih, Greer Swinard, Kate Lievesley, Kayley O'Flynn, Suzi Marquis, Rebecca Whittemore, Xiaohui $\mathrm{Xu}$, and everyone on the TEDS team.

Conflict of Interest For Philip Asherson: honoraria paid to King's College London research and educational support funds for consultancy, speaking at sponsored events, educational support or research for Shire, Eli-Lilly, Novartis, Jannssen, Vifor Pharma, GW Pharma and QbTech.

\section{References}

American Psychiatric Association. (2000). Diagnostic and statistical manual of mental disorders, 4th edition, text revision (4th ed.). Washington: APA.

American Psychiatric Association. (2013). Diagnostic and statistical manual of mental disorders, 5th edition (5th ed.). Arlington: Author.

Andreou, P., Neale, B. M., Chen, W., Christiansen, H., Gabriels, I., Heise, A., et al. (2007). Reaction time performance in ADHD: improvement under fast-incentive condition and familial effects. Psychological Medicine, 37, 1703-1715.

Borger, N., \& van der Meere, J. J. (2000). Motor control and state regulation in children with ADHD: a cardiac response study. Biological Psychiatry, 51, 247-267.

Buhler, E., Bachmann, C., Goyert, H., Heinzel-Gutenbrunner, M., \& Kamp-Becker, I. (2011). Differential diagnosis of autism spectrum disorder and attention deficit hyperactivity disorder by means of inhibitory control and "theory of mind". Journal of Autism and Developmental Disorders, 41, 1718-1726.

Chen, W., Zhou, K., Sham, P., Franke, B., Kuntsi, J., Campbell, D., et al. (2008). DSM-IV combined type ADHD shows familial association with sibling trait scores: a sampling strategy for QTL linkage. American Journal of Medical Genetics Part B: Neuropsychiatric Genetics, 147B, 1450-1460.

Conners, C. K., Sitarenios, G., Parker, J. D., \& Epstein, J. N. (1998a). The revised Conners' parent rating scale (CPRS-R): factor structure, reliability, and criterion validity. Journal of Abnormal Child Psychology, 26, 257-268.

Conners, C. K., Sitarenios, G., Parker, J. D., \& Epstein, J. N. (1998b). Revision and restandardization of the Conners teacher rating scale 
(CTRS-R): factor structure, reliability, and criterion validity. Journal of Abnormal Child Psychology, 26, 279-291.

Constantino, J. N., Hudziak, J. J., \& Todd, R. D. (2003). Deficits in reciprocal social behavior in male twins: evidence for a genetically independent domain of psychopathology. Journal of the American Academy of Child and Adolescent Psychiatry, 42, 458-467.

Cross-Disorder Group of the Psychiatric Genomics Consortium. (2013). Genetic relationship between five psychiatric disorders estimated from genome-wide SNPs. Nature Genetics, 45, 984-994.

Dawson, G., Webb, S., Schellenberg, G. D., Dager, S., Friedman, S., Aylward, E., \& Richards, T. (2002). Defining the broader phenotype of autism: genetic, brain, and behavioral perspectives. Development and Psychopathology, 14, 581-611.

Dworzynski, K., Happe, F., Bolton, P., \& Ronald, A. (2009). Relationship between symptom domains in autism spectrum disorders: a population based twin study. Journal of Autism and Developmental Disorders, 39, 1197-1210.

Geurts, H. M., Verte, S., Oosterlaan, J., Roeyers, H., \& Sergeant, J. A. (2004). How specific are executive functioning deficits in attention deficit hyperactivity disorder and autism? Journal of Child Psychology and Psychiatry, 45, 836-854.

Geurts, H. M., Grasman, R. P., Verte, S., Oosterlaan, J., Roeyers, H., van Kammen, S. M., \& Sergeant, J. A. (2008). Intra-individual variability in ADHD, autism spectrum disorders and Tourette's syndrome. Neuropsychologia, 46, 3030-3041.

Greven, C. U., Rijsdijk, F. V., \& Plomin, R. (2011). A twin study of ADHD symptoms in early adolescence: hyperactivity-impulsivity and inattentiveness show substantial genetic overlap but also genetic specificity. Journal of Abnormal Child Psychology, 39, 265-275.

Happe, F., Booth, R., Charlton, R., \& Hughes, C. (2006). Executive function deficits in autism spectrum disorders and attention-deficit/ hyperactivity disorder: examining profiles across domains and ages. Brain and Cognition, 61, 25-39.

Johnson, K. A., Robertson, I. H., Kelly, S. P., Silk, T. J., Barry, E., Daibhis, A., et al. (2007). Dissociation in performance of children with ADHD and high-functioning autism on a task of sustained attention. Neuropsychologia, 45, 2234-2245.

Kuntsi, J., \& Klein, C. (2012). Intraindividual variability in ADHD and its implications for research of causal links. Current Topics in Behavioral Neurosciences, 9, 67-91.

Kuntsi, J., Andreou, P., Ma, J., Borger, N. A., \& van der Meere, J. J. (2005). Testing assumptions for endophenotype studies in ADHD: reliability and validity of tasks in a general population sample. $B M C$ Psychiatry, 5, 40.

Kuntsi, J., Rogers, H., Swinard, G., Borger, N., van der Meere, J., Rijsdijk, F., \& Asherson, P. (2006). Reaction time, inhibition, working memory and 'delay aversion' performance: genetic influences and their interpretation. Psychological Medicine, 36, 1613-1624.

Kuntsi, J., Wood, A. C., Rijsdijk, F., Johnson, K. A., Andreou, P., Albrecht, B., et al. (2010). Separation of cognitive impairments in attention-deficit/hyperactivity disorder into 2 familial factors. Archives of General Psychiatry, 67, 1159-1167.

Kuntsi, J., Pinto, R., Price, T. S., van der Meere, J. J., Frazier-Wood, A. C., \& Asherson, P. (2014). The separation of ADHD inattention and hyperactivity-impulsivity symptoms: pathways from genetic effects to cognitive impairments and symptoms. Journal of Abnormal Child Psychology, 42, 127-136.

Levy, Y., \& Ebstein, R. P. (2009). Research review: crossing syndrome boundaries in the search for brain endophenotypes. Journal of Child Psychology and Psychiatry, 50, 657-668.

Loehlin, J. C. (1996). The Cholesky approach: a cautionary note. Behavior Genetics, 26, 65-69.

Losh, M., Sullivan, P. F., Trembath, D., \& Piven, J. (2008). Current developments in the genetics of autism: from phenome to genome. Journal of Neuropathology and Experimental Neurology, 67, 829-837.
Lubke, G. H., Hudziak, J. J., Derks, E. M., van Bijsterveldt, T. C., \& Boomsma, D. I. (2009). Maternal ratings of attention problems in ADHD: evidence for the existence of a continuum. Journal of the American Academy of Child and Adolescent Psychiatry, 48, 1085-1093.

Lundstrom, S., Chang, Z., Kerekes, N., Gumpert, C. H., Rastam, M., Gillberg, C., et al. (2011). Autistic-like traits and their association with mental health problems in two nationwide twin cohorts of children and adults. Psychological Medicine, 41, 2423-2433.

McLoughlin, G., Ronald, A., Kuntsi, J., Asherson, P., \& Plomin, R. (2007). Genetic support for the dual nature of attention deficit hyperactivity disorder: substantial genetic overlap between the inattentive and hyperactive-impulsive components. Journal of Abnormal Child Psychology, 35, 999-1008.

Meyer-Lindenberg, A. (2010). Intermediate or brainless phenotypes for psychiatric research? Psychological Medicine, 40, 1057-1062.

Neale, M. C., Boker, S. M., Xie, G., \& Maes, H. (2006). Mx: statistical modeling (7th ed.). Richmond: Department of Psychiatry.

Nyden, A., Gillberg, C., Hjelmquist, E., \& Heiman, M. (1999). Executive function/attention deficits in boys with Asperger syndrome, attention disorder and reading/writing disorder. Autism, 3, 213-228.

Nyden, A., Niklasson, L., Stahlberg, O., Anckarsater, H., Wentz, E., Rastam, M., \& Gillberg, C. (2010). Adults with autism spectrum disorders and ADHD neuropsychological aspects. Research in Developmental Disabilities, 31, 1659-1668.

Polderman, T. J. C., Hoekstra, R. A., Posthuma, D., \& Larsson, H. (2014). The co-occurrence of autistic and ADHD dimensions in adults: an etiological study in 17770 twins. Translational Psychiatry, 4, e435. doi:10.1038/tp.2014.84.

Posthuma, D., \& Polderman, T. J. C. (2013). What have we learned from recent twin studies about the etiology of neurodevelopmental disorders? Current Opinion in Neurology, 26, 111-121.

Price, T. S., Freeman, B., Craig, I., Petrill, S. A., Ebersole, L., \& Plomin, R. (2000). Infant zygosity can be assigned by parental report questionnaire data. Twin Research and Human Genetics, 3, 129-133.

Raymaekers, R., Antrop, I., van der Meere, J. J., Wiersema, J. R., \& Roeyers, H. (2007). HFA and ADHD: a direct comparison on state regulation and response inhibition. Journal of Clinical and Experimental Neuropsychology, 29, 418-427.

Reiersen, A. M., Constantino, J. N., Grimmer, M., Martin, N. G., \& Todd, R. D. (2008). Evidence for shared genetic influences on selfreported ADHD and autistic symptoms in young adult Australian twins. Twin Research and Human Genetics, 11, 579-585.

Rijsdijk, F. V., \& Sham, P. C. (2002). Analytic approaches to twin data using structural equation models. Briefings in Bioinformatics, 3, 119-133.

Robinson, E. B., Koenen, K. C., McCormick, M. C., Munir, K., Hallett, V., Happe, F., et al. (2011). Evidence that autistic traits show the same etiology in the general population and at the quantitative extremes $(5 \%, 2.5 \%$, and $1 \%)$. Archives of General Psychiatry, 68 , $1113-1121$.

Robinson, E. B., Koenen, K. C., McCormick, M. C., Munir, K., Hallett, V., Happe, F., et al. (2012). A multivariate twin study of autistic traits in 12-year-olds: testing the fractionable autism triad hypothesis. Behavior Genetics, 42, 245-255.

Rommelse, N. N. J., Franke, B., Geurts, H. M., Hartman, C. A., \& Buitelaar, J. K. (2010). Shared heritability of attention-deficit/hyperactivity disorder and autism spectrum disorder. European Child \& Adolescent Psychiatry, 19, 281-295.

Rommelse, N. N. J., Geurts, H. M., Franke, B., Buitelaar, J. K., \& Hartman, C. A. (2011). A review on cognitive and brain endophenotypes that may be common in autism spectrum disorder and attention-deficit/hyperactivity disorder and facilitate the search for pleiotropic genes. Neuroscience and Biobehavioral Reviews, 35 , 1363-1396. 
Ronald, A., \& Hoekstra, R. A. (2011). Autism spectrum disorders and autistic traits: a decade of new twin studies. American Journal of Medical Genetics Part B: Neuropsychiatric Genetics, 156, 255-274.

Ronald, A., Happe, F., \& Plomin, R. (2005). The genetic relationship between individual differences in social and nonsocial behaviours characteristic of autism. Developmental Science, 8, 444-458.

Ronald, A., Happe, F., Bolton, P., Butcher, L. M., Price, T. S., Wheelwright, S., et al. (2006a). Genetic heterogeneity between the three components of the autism spectrum: a twin study. Journal of the American Academy of Child and Adolescent Psychiatry, 45, 691-699.

Ronald, A., Happe, F., Price, T. S., Baron-Cohen, S., \& Plomin, R. (2006b). Phenotypic and genetic overlap between autistic traits at the extremes of the general population. Journal of the American Academy of Child and Adolescent Psychiatry, 45, 1206-1214.

Ronald, A., Simonoff, E., Kuntsi, J., Asherson, P., \& Plomin, R. (2008). Evidence for overlapping genetic influences on autistic and ADHD behaviours in a community twin sample. Journal of Child Psychology and Psychiatry, 49, 535-542.

Ronald, A., Butcher, L. M., Docherty, S., Davis, O. S., Schalkwyk, L. C., Craig, I. W., \& Plomin, R. (2010a). A genome-wide association study of social and non-social autistic-like traits in the general population using pooled DNA, $500 \mathrm{~K}$ SNP microarrays and both community and diagnosed autism replication samples. Behavior Genetics, 40, 31-45.

Ronald, A., Edelson, L. R., Asherson, P., \& Saudino, K. J. (2010b). Exploring the relationship between autistic-like traits and ADHD behaviors in early childhood: findings from a community twin study of 2-year-olds. Journal of Abnormal Child Psychology, 38, $185-196$.

Ronald, A., Larsson, H., Anckarsater, H., \& Lichtenstein, P. (2011). A twin study of autism symptoms in Sweden. Molecular Psychiatry, 16, 1039-1047.

Ronald, A., Larsson, H., Anckarster, H., \& Lichtenstein, P. (2014). Symptoms of autism and ADHD: A Swedish twin study examining their overlap. Journal of Abnormal Psychology, 123, 440-51.

Sattler, J. M. (1992). Assessment of children: WAIC-III and WPPSI-R Supplement. San Diego: Jerome M Sattler.

Scott, F. J., Baron-Cohen, S., Bolton, P., \& Brayne, C. (2002). The CAST (Childhood Asperger Syndrome Test): preliminary development of a UK screen for mainstream primary-school-age children. Autism, 6, 9-31.

Simonoff, E., Pickles, A., Charman, T., Chandler, S., Loucas, T., \& Baird, G. (2008). Psychiatric disorders in children with autism spectrum disorders: prevalence, comorbidity, and associated factors in a population-derived sample. Journal of the American Academy of Child and Adolescent Psychiatry, 47, 921-929.

Sinzig, J., Morsch, D., Bruning, N., Schmidt, M. H., \& Lehmkuhl, G. (2008). Inhibition, flexibility, working memory and planning in autism spectrum disorders with and without comorbid ADHD-symptoms. Child and Adolescent Psychiatry and Mental Health, 2, 4.

Stata. (2007). Stata statistical software release 9.0: survey data manual. College Station: Stata Corporation.

Taylor, M. J., Charman, T., Robinson, E. B., Plomin, R., Happe, F., Asherson, P., \& Ronald, A. (2013). Developmental associations between traits of autism spectrum disorder and attention deficit hyperactivity disorder: a genetically informative, longitudinal twin study. Psychological Medicine, 43, 1735-1746.

Taylor, M. J., Charman, T., \& Ronald, A. (2015). Where are the strongest associations between autistic traits and traits of ADHD? Evidence from a community-based twin study. European Child and Adolescent Psychiatry. doi:10.1007/s00787-014-0666-0.

Tye, C., Asherson, P., Ashwood, K. L., Azadi, B., Bolton, P., \& McLoughlin, G. (2014). Attention and inhibition in children with ASD, ADHD and co-morbid ASD+ADHD: an event-related potential study. Psychological Medicine, 44, 1101-1116.

van der Meere, J. J., Stemerdink, N., \& Gunning, B. (1995). Effects of presentation rate of stimuli on response inhibition in ADHD children with and without tics. Perceptual and Motor Skills, 81, 259-262.

Viding, E., \& Blakemore, S. J. (2007). Endophenotype approach to developmental psychopathology: implications for autism research. Behavior Genetics, 37, 51-60.

Wechsler, D. (1991). Wechsler intelligence scale for children (3rd ed.). London: Psychological Corporation.

Willcutt, E. G., Doyle, A. E., Nigg, J. T., Faraone, S. V., \& Pennington, B. F. (2005). Validity of the executive function theory of attentiondeficit/hyperactivity disorder: a meta-analytic review. Biological Psychiatry, 57, 1336-1346.

Wood, A. C., Asherson, P., van der Meere, J. J., \& Kuntsi, J. (2010). Separation of genetic influences on attention deficit hyperactivity disorder symptoms and reaction time performance from those on IQ. Psychological Medicine, 40, 1027-1037.

Wood, A. C., Rijsdijk, F., Johnson, K. A., Andreou, P., Albrecht, B., Arias-Vasquez, A., et al. (2011). The relationship between ADHD and key cognitive phenotypes is not mediated by shared familial effects with IQ. Psychological Medicine, 41, 861-871. 